\title{
The $\Delta a$ research group
}

\section{H.M. Maitzen, E. Paunzen, H. Pöhnl, M. Rode-Paunzen, M. Netopil, Ch. Stütz and H. Baum}

Institut für Astronomie, Universität Wien, Türkenschanzstrasse 17, 1180 Vienna, Austria email: maitzen@astro.univie.ac.at

\begin{abstract}
We summarize of more than 25 years of research with the three filter, intermediateband, $\Delta a$ photometric system. It investigates the flux depression at $\lambda 5200$ found in magnetic chemically peculiar (CP) objects. Starting with photoelectric measurements it has steadily developed introducing new and more efficient filters as well as the modern CCD technique. So far more than twenty papers were devoted to searching for new CP stars in our Milky Way up to distances of $5000 \mathrm{pc}$ and even in the Large Magellanic Cloud. In the latter, the first extragalactic CP stars were detected. In addition, we have presented theoretical isochrones and synthetic colors from the latest available stellar atmospheres. The theoretical predictions agree very well with observations allowing not only to determine the reddening and age of open clusters from our photometry but also to investigate the flux depression at $\lambda 5200$ in more detail. As an outlook, we present a new approach to search for chemically peculiar horizontal branch stars in globular clusters and to detect stellar variability of various objects observed during our photometric observations.
\end{abstract}

Keywords. Stars: chemically peculiar, stars: magnetic fields, stars: evolution, techniques: photometric, galaxies: star clusters

\section{The tool of $\Delta a$-photometry}

Photometry offers an way for the investigation of large samples of CP stars, especially through the discovery of characteristic broad band absorption features, the most suitable of them located around $\lambda 5200$. Nearly three decades ago, Maitzen (1976) introduced the narrow-band, three filter $\Delta a$ photometric system, slightly changed within the last years, to investigate the flux depression at $\lambda 5200$. It samples the depth of this flux depression by comparing the flux at the center $\left(\lambda 5205-g_{2}\right)$, with the adjacent regions $\left(\lambda 5027-g_{1}\right.$ and $\lambda 5509-y)$ using bandpasses of $222 \AA\left(g_{1}\right), 107 \AA\left(g_{2}\right)$ and $120 \AA(y)$. The respective index was introduced as:

$$
a=g_{2}-\frac{g_{1}+y}{2}
$$

Since this quantity is slightly dependent on temperature (increasing towards lower temperatures), the intrinsic peculiarity index had to be defined as $\Delta a=a-a[(b-$ $y) ;(B-V) ;\left(g_{1}-y\right)$ ] (the difference between the individual a-values and the a-value of non-peculiar stars of the same colour - the "normality line"). It was shown (e.g., Vogt et al. 1998) that virtually all peculiar stars with magnetic fields (CP2 stars) have positive $\Delta a$-values up to 0.075 mag whereas $\mathrm{Be} / \mathrm{Ae}$ and $\lambda$ Bootis stars exhibit significant negative ones (Maitzen \& Pavlovski 1989a, 1989b). The index $\left(g_{1}-y\right)$ shows an excellent correlation with $(b-y)$ and can be used as an index for the effective temperature. A $y$ versus $\left(g_{1}-y\right)$ diagram (= color-magnitude diagram) for a cluster can then be easily used to sort out nonmembers. Assuming that all stars exhibit the same interstellar reddening, peculiar objects deviate from the normality line more than $3 \sigma$ (see Figure 1). 

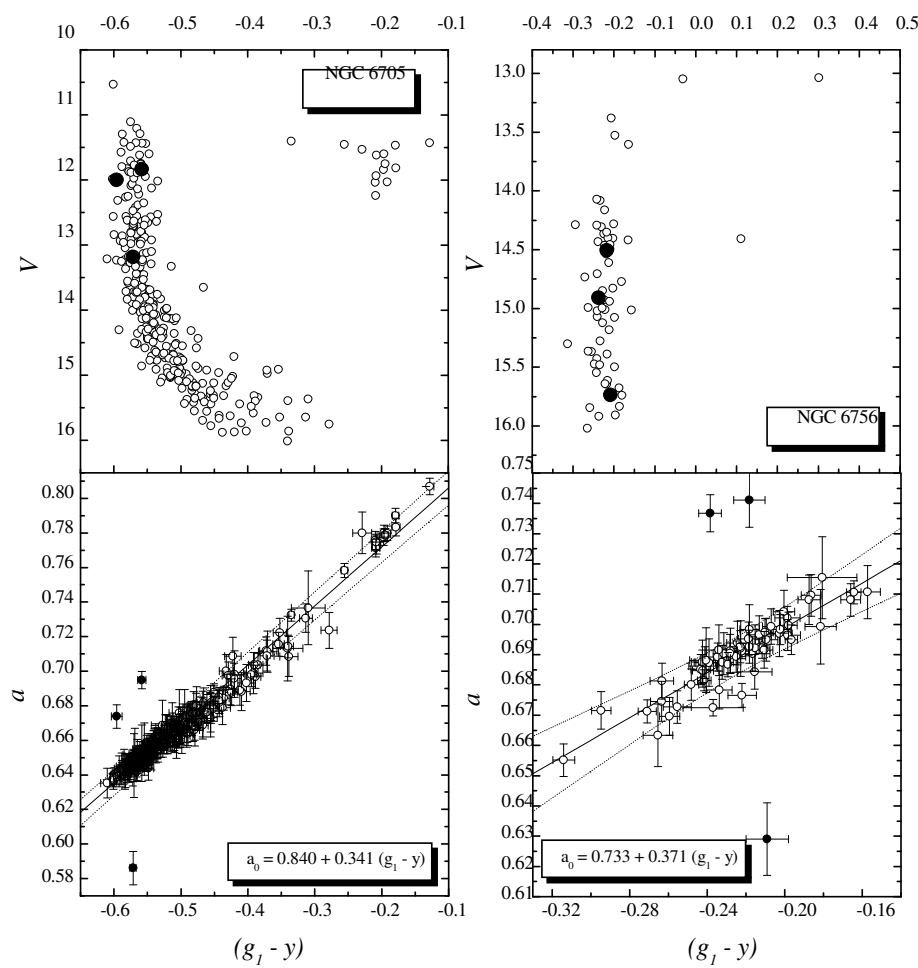

Figure 1. The $a$-Index versus $\left(g_{1}-y\right)$ and the color-magnitude diagrams for the open clusters NGC 6705 and NGC 6756 . The full line is the normality line, the dotted lines are the confidence intervals $(3 \sigma)$. Three peculiar objects were found, two with significant positive $\Delta a$-values, one with a negative one (Paunzen et al. 2003).
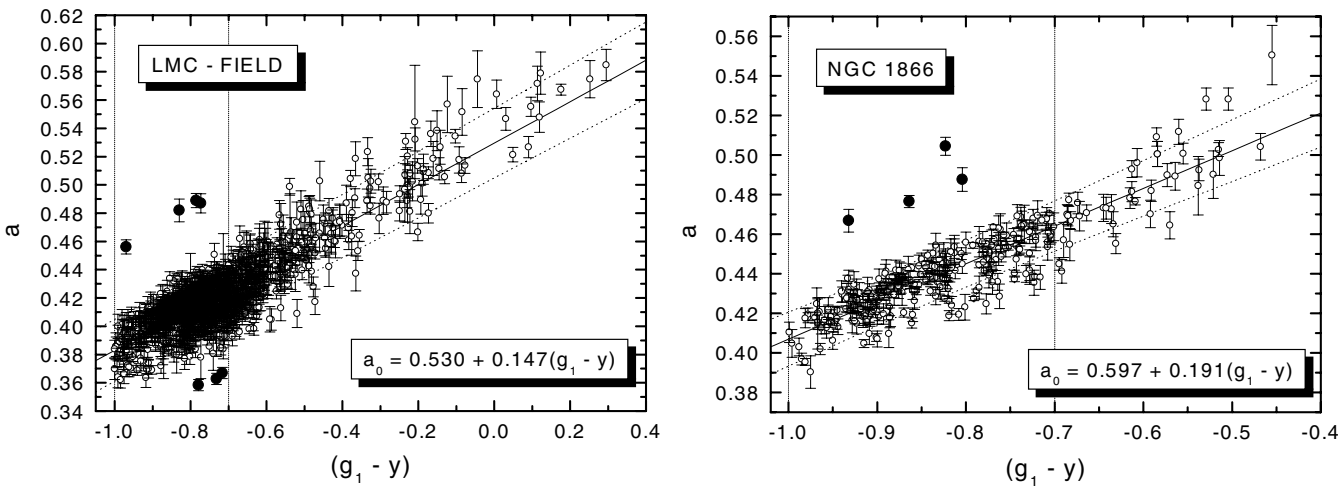

Figure 2. The $a$-Index versus $\left(g_{1}-y\right)$ for NGC 1866 (right panel) and its surrounding (left panel). Filled circles indicate apparent peculiar stars. The vertical lines denote the spectral type range (B8 to F2) where chemical peculiar stars are expected.

\section{Open cluster survey}

The survey for CP stars in open clusters using photoelectric photometry (one star at one time) has so far delivered data for objects usually no more distant than $1000 \mathrm{pc}$ from the Sun. A series of fourteen papers (first: Maitzen \& Hensberge (1981); for the time being last: Maitzen (1993)) were devoted to 1240 stars in 38 open cluster fields with the 


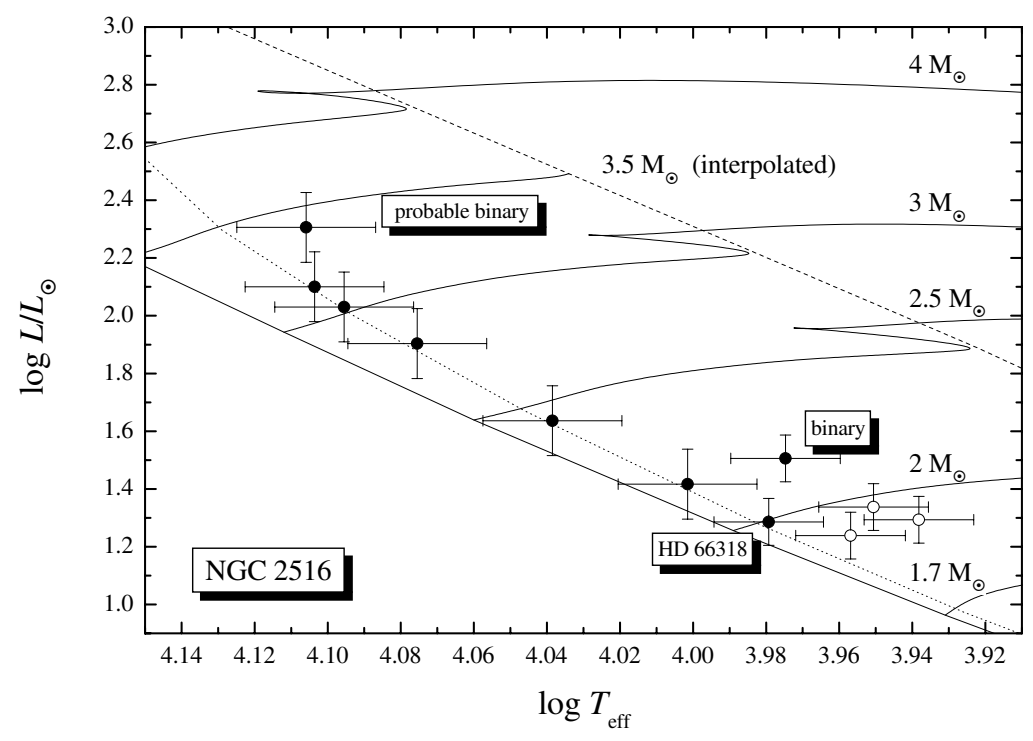

Figure 3. The location of the CP2 stars for NGC 2516. Filled circles are well established CP2 objects and open circles are doubtful cases. The dotted line is the isochrone for $\log t=8.00$ and $[\mathrm{Fe} / \mathrm{H}]=-0.23$ whereas the dashed line denoted the Terminal Age Main Sequence. A strong magnetic field in HD 66318 was discovered by Bagnulo et al. (2003).

detection of $62 \mathrm{CP}$ candidates. In 1995 a survey in open clusters using CCD technology was initialized. So far $\Delta a$-photometry of 23 open clusters wre published in a series of 5 papers (Bayer et al. 2000, Paunzen \& Maitzen 2001, 2002, Paunzen et al. 2002, 2003). Within the investigated sample of nearly 2600 stars, more than $30 \mathrm{CP}$ candidates were found.

Our search for new variable stars in open clusters is a serendipitous result from already published CCD $\Delta a$-photometry (Paunzen et al. 2004). The observations span widely different time intervals (0.02 to 60 days) yielding different possibilities for detecting the whole set of variations. We want to emphasize that these observations are not optimized for the detection of variable stars but are able to find even very low amplitude variables (the typical detection limit reached is between 0.006 and $0.022 \mathrm{mag}$ ). In total, we have detected 35 variable objects of which four are not members of their corresponding clusters. The variables cover the entire Hertzsprung-Russell diagram.

\section{Extragalactic CP stars}

The first extragalactic classical chemically peculiar stars more than 100 years after the discovery of this group were detected (Maitzen et al. 2001). Using CCD $\Delta a$-photometry, eight definite positive detections in the globular-like stellar cluster NGC 1866 and its surroundings in the Large Magellanic Cloud were made (Figure 2). More than 1950 objects down to $20.5 \mathrm{mag}$ were observed. Furthermore we found three objects with significantly negative $\Delta a$-values indicating either an Ae/shell or $\lambda$ Bootis nature. The overall percentage of chemically peculiar stars for NGC 1866 (1.5\%) seems lower than for the majority of open clusters in our Milky Way whereas the incidence within the LMC field is decisively less $(0.3 \%)$. 


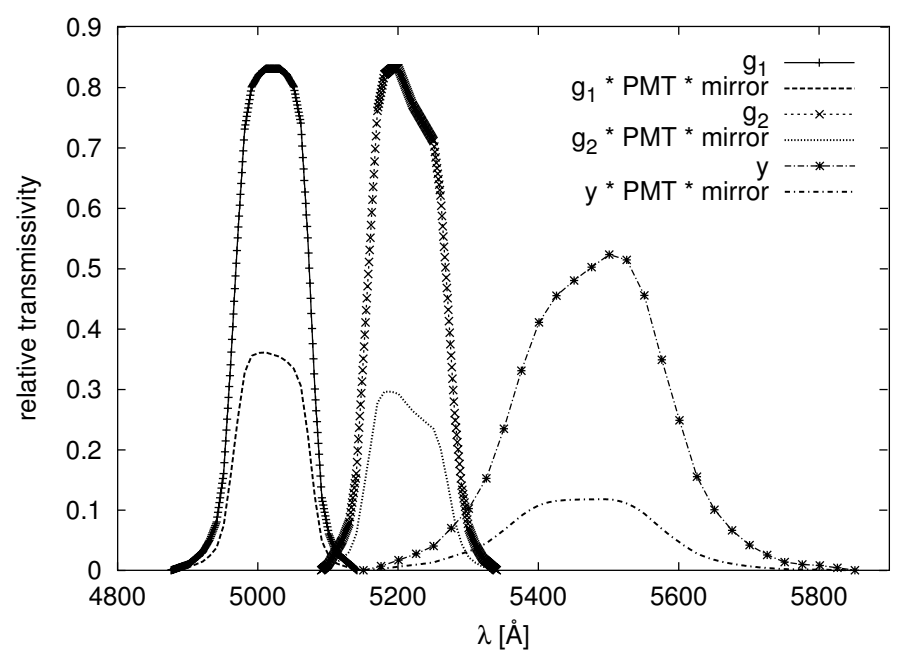

Figure 4. Filter transmission functions and effective transmission of the synthetic photometric system after convolution with the profiles of the response function of a photomultiplier tube and a typical mirror reflection efficiency function (Kupka et al. 2003).

\section{Evolutionary status of CP stars of the upper Main Sequence}

For many decades the evolutionary status of the CP stars was controversial. Gómez et al. (1998) presented a Hertzsprung-Russell-diagram of about $1000 \mathrm{CP}$ stars in the Solar Neighbourhood using astrometric data from the Hipparcos satellite as well as photometric and radial velocity data. Most CP stars lie on the Main Sequence occupying the whole width of it (about $2 \mathrm{mag}$ ), just like "normal" stars in the same range of spectral types. Hubrig et al. (2000) challenged these results and found that the distribution of the magnetic CP stars of masses below $3 \mathrm{M}_{\odot}$ in the Hertzsprung-Russell-diagram differs from that of the "normal" stars in the same temperature range at a high level of significance. Magnetic stars are concentrated toward the center of the Main Sequence band. In particular, they found that magnetic fields appear only in stars which have already completed at least approximately 30\% of their Main Sequence life time. The somewhat discrepant results might be understood by the selection of objects. The detection of resolved Zeeman patterns requires a specifically slow rotation. This gives preference to finding such objects in advanced phases on the Main Sequence band where rotational velocities have been decreased by the growth of stellar radii.

We have investigated four young (age $\leqslant 100 \mathrm{Myr}$ ) open clusters with known CP2 members (see Figure 3 for NGC 2516). The peculiarity of these objects was established via photometric as well as spectroscopic data. We have derived effective temperatures and luminosities for these objects and calibrated ages as well as masses with the help of standard evolutionary models taking the overall metallicities of the individual clusters into account.

All investigated objects are members of open clusters with ages between 10 and 140 Myr corresponding to $0.05 \%$ and $0.36 \%$ of their Main Sequence lifetimes, respectively. This clearly proves that the observable CP2 phenomenon occurs already well before a star has reached $30 \%$ of its lifetime on the Main Sequence (Pöhnl et al. 2003) 
M 30 (794 stars, 46 frames)

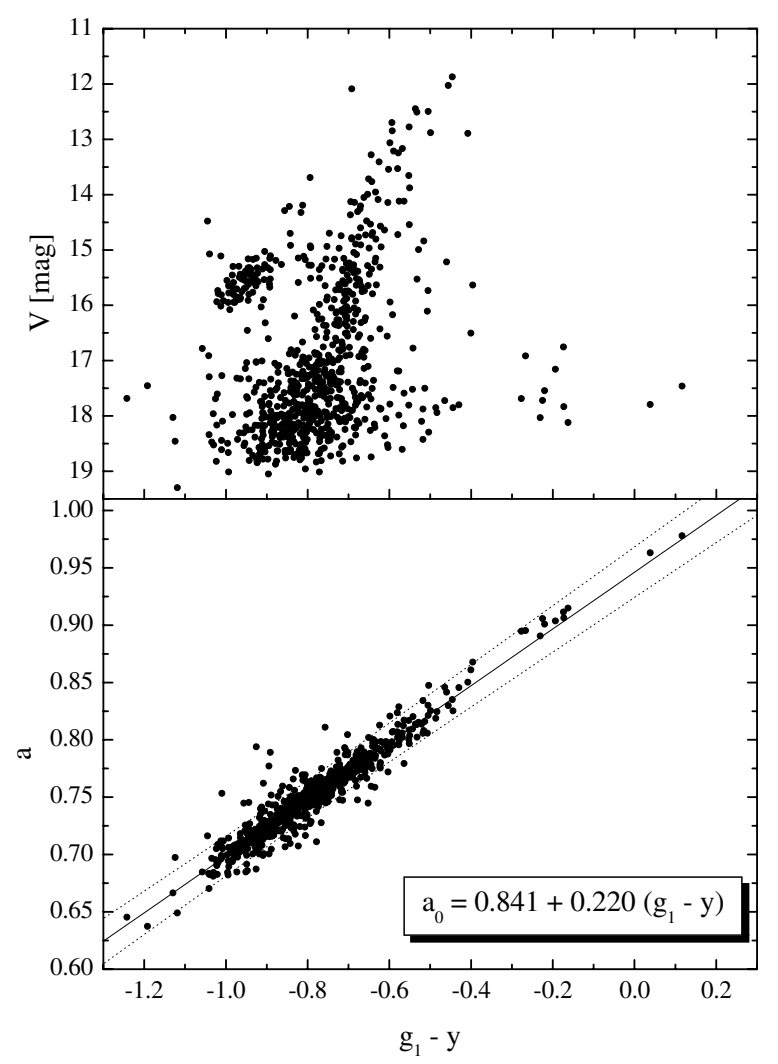

Figure 5. The $a$-Index versus $\left(g_{1}-y\right)$ and the color-magnitude diagram for the globular cluster M30. Some peculiar stars were found.

\section{Theoretical aspects}

A synthetic photometric system (Figure 4) was developed by Kupka et al. (2003) that can be used to explore the capability of model atmospheres with individual elemental abundances to predict photometric $\Delta a$ magnitudes. The observed dependency of the $a$-index as a function of various colour indices sensitive to the effective temperature of stars as well as its average scatter expected from surface gravity variations within the main-sequence band is confirmed, also the behaviour of the normality line is well reproduced. Kupka et al. (2003) have also shown the metallicity dependence of the $a$-Index. Thus, $\Delta a$ photometry is a viable tool to identify CP stars in samples with metallicities slightly different from the solar ones and it is well suited to draw statistically meaningful conclusions about their distribution.

Kupka et al. (2004) presented model atmospheres computed with individual abundances for a representative sample of CP stars and either confirmed or redetermined their input parameters through comparisons with photometric, spectrophotometric and high resolution spectroscopic data. The final models obtained from this procedure were used to compute synthetic $\Delta a$ indices which were compared with observations. We are able to reproduce the observed $\Delta a$ values for CP1 (almost zero), cool CP2 (positive) and $\lambda$ Bootis stars (negative) within a few mmags. 
In addition Claret et al. (2003) have investigated the capability of theoretical isochrones for the photometric $\Delta a$ system to derive astrophysical parameters such as age, reddening and distance modules for open clusters. As a test, the published data of 23 open clusters were used to fit these isochrones with parameters from the literature. The fits show an excellent agreement between the observations and the theoretical grid. The observational data have been also fitted without knowledge of age, reddening and distance modulus, yielding an accuracy of 5 to $15 \%$ depending on the well known error sources of such a method.

\section{A new approach}

The existence of peculiar stars on the Blue Horizontal Branch was already shown by Behr et al. (1999). They used high resolution spectra to determine the elemental abundances of individual objects to prove the peculiarities. Our technique is capable of covering simultaneously all members of a globular cluster. A preliminary result of our photometric survey of M 30, where a few peculiar stars were found with the aid of $\Delta a$ photometry, is shown in Figure 5.

Another capability of the $\Delta a$ tool is to detect Novae. Only a small sample was investigated up to now, but the symbiotic nova PU Vulpeculae as well as the dwarf nova Z Cam show significant negative $\Delta a$-values up to 0.1 mag. Further observations on that topic are planned at the Figl-Observatory (Austria).

\section{References}

Bagnulo, S., Landstreet, J.D., Lo Curto, G., Szeifert, T., Wade, G.A. 2003, A\&A 403, 645

Bayer, C., Maitzen, H.M., Paunzen, E., Rode-Paunzen, M., Sperl, M. 2000, A $\varangle A S$ 147, 99

Behr, B.B., Cohen, J.G., McCarthy, J.K., Djorgovski, S.G. 1999, ApJ 517, 135

Claret, A., Paunzen, E., Maitzen, H.M. 2003, A 6 A 412, 91

Gómez, A.E., Luri, X., Grenier, S., Figueras, F., North, P., Royer, F., Torra, J., Mennessier, M.O. 1998, A\&̈A 336, 953

Hubrig, S., North, P., Mathys, G. 2000, ApJ 539, 352

Kupka, F., Paunzen, E., Iliev, I.Kh., Maitzen, H.M. 2004, MNRAS 352, 863

Kupka, F., Paunzen, E., Maitzen, H.M. 2003, MNRAS 341, 849

Maitzen, H.M. 1976, A\&\&A 51, 223

Maitzen, H.M. 1993, A\&AS 102, 1

Maitzen, H.M., Hensberge, H. 1981, A\&A 96, 151

Maitzen, H.M., Paunzen, E., Pintado, O.I. 2001, A\&A 371, L5

Maitzen, H.M., Pavlovski, K. 1989a, A $\mathscr{E} A S$ 77, 351

Maitzen, H.M., Pavlovski, K. 1989a, A\&A 219, 253

Paunzen, E., Maitzen, H.M. 2001, A\&A 373, 153

Paunzen, E., Maitzen, H.M. 2002, A\&A 385, 867

Paunzen, E., Pintado, O.I., Maitzen, H.M. 2002, A $\& A$ 395, 823

Paunzen, E., Pintado, O.I., Maitzen, H.M. 2003, A\& $A$ 412, 721

Paunzen, E., Zwintz, K., Maitzen, H.M., Pintado, O.I., Rode-Paunzen, M. 2004, A\&A 418, 99

Pöhnl, H., Maitzen, H.M., Paunzen, E. 2003, A\&\&A 402, 247

Vogt, N., Kerschbaum, F., Maitzen, H.M., Faundez-Abans, M. 1998, A $\& A S$ 130, 455 